\title{
Ultrafast dynamics of water at the water-air interface studied by femtosecond surface vibrational spectroscopy
}

\author{
Mischa Bonn ${ }^{1,2}$, Cho-Shuen Hsieh'1, Lukasz Piatkowski², Huib J. Bakker ${ }^{2}$ and Zhen Zhang1 \\ 1 Max Planck Institute for Polymer Research, Ackermannweg 10, 55128 Mainz, Germany \\ 2 FOM-Institute AMOLF, Science Park 104, 1098 XG Amsterdam, The Netherlands
}

\begin{abstract}
We study the dynamics of water molecules at the water-air interface, using surfacespecific two-dimensional infrared sum-frequency generation (2D-SFG) spectroscopy. The data reveal the occurrence of surprisingly fast energy transfer and reorientational dynamics at aqueous interfaces.
\end{abstract}

\section{Introduction}

Water is very different from liquids of similar molecular weight and one of its unique properties is the very efficient transfer of vibrational energy between molecules, which is on account of strong dipoledipole interactions between the $\mathrm{O}-\mathrm{H}$ oscillators. While we have a sound understanding of such energy transfer in bulk water we know less about how and how quickly transfer occurs at its interface with a hydrophobic phase because of the challenge of specifically addressing the outermost monolayer.

In bulk water, energy flow mechanisms and dynamics have been quantified using femtosecond infrared laser techniques. The $\mathrm{O}-\mathrm{H}$ stretch vibrations of the water molecules were found to show rapid resonant (Förster) energy transfer on a time scale $<100 \mathrm{fs}$, largely as a result of the strong dipole-dipole interactions between the $\mathrm{O}-\mathrm{H}$ oscillators. Reorientation of water in bulk has been found to occur on a timescale of $\sim 2.5$ ps at room temperature ${ }^{1}$, through a hydrogen-bond jump mechanism ${ }^{2}$.

The question that presents itself is: how do the local structural changes affect the mechanism and timescale of energy flow and reorientation at an aqueous interface? The challenge of characterizing the structure of the outermost surface molecules has been met by sum frequency generation (SFG) spectroscopy of their O-H stretch vibrations. In an SFG experiment, infrared and visible laser pulses are overlapped in space and time on the surface, and the sum-frequency of the two laser fields can be generated, but only in the surface region. If the infrared light is resonant with the $\mathrm{O}-\mathrm{H}$ stretch vibration of surface water, this process is resonantly enhanced. Initial SFG studies, making use of the distinct correlation that exists between the local hydrogen bonding strength and the $\mathrm{O}-\mathrm{H}$ stretch vibrational frequency of an $\mathrm{O}-\mathrm{H}$ group in a water molecule, indicated that, in addition to the 'free' $\mathrm{O}-\mathrm{H}$ groups pointing away from the surface, different types of hydrogen-bonded interfacial water also exist and that the interfacial layer is limited to $\sim 1$ monolayer.

\section{Energy flow dynamics}

We evaluate interfacial energy flow dynamics, using surface-specific two-dimensional infrared sumfrequency generation (2D-SFG) spectroscopy 3,4 . This technique combines the unique capabilities of 2D-IR spectroscopy with the surface specificity and (sub-) monolayer sensitivity of SFG spectroscopy. It enables new insights into energy transfer at water interfaces by investigating the effect of widely tuneable (2100$3000 \mathrm{~cm}^{-1}$ ) excitation pulses of limited spectral width (typically set at $100 \mathrm{~cm}^{-1}$ ) on the SFG response of interfacial water molecules. The 2D-IR SFG data (see, e.g. Fig. 1) reveal the occurrence of surprisingly fast intra- and intermolecular energy transfer processes at aqueous interfaces. The results show that energy transfer among OD stretching modes of water on the surface is rapid and efficient and that OD excitations move both along the surface plane and down into the bulk ${ }^{5}$. . Such information on interfacial aqueous energy flow patterns contribute to our understanding of chemistry at aqueous interfaces, as chemical reactions at aqueous surfaces, be it on catalytic water interfaces, atmospheric chemistry or at cell membrane surfaces.

\section{Reorientational Dynamics}

We probe the motion of free $\mathrm{OH}$ groups at the air/water interface using time- and polarization-resolved IR pump / vibrational sum frequency probe measurements ${ }^{7}$. The pump pulse induces vibrational excitation for molecules oriented preferentially along the polarization axis of the pump field. 


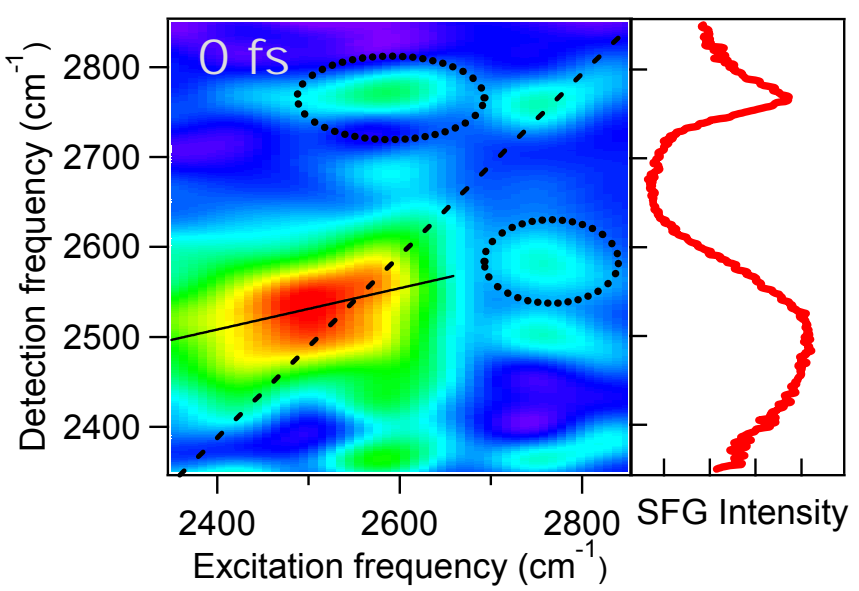

Fig. 1. Time-resolved 2D-SFG data for interfacial water at the water/air interface at zero delay time between pump and probe. The right panel shows the static SFG spectrum. The 2D spectrum reveals an on-diagonal response with a slope smaller than one (dashed line indicates slope of 1), expected for a heterogeneous surface. Off-diagonal peaks revealing energy transfer are highlighted by dotted ovals.

These time-resolved VSF spectroscopic results with multiple pump polarizations and an appropriate theoretical model, provides insights into interfacial reorientational motion. The reorientation of the free $\mathrm{OH}$ is found to be three times faster than the reorientation of hydrogen bonded $\mathrm{OH}$ groups in bulk, due to the lower degree of hydrogen bond coordination at the interface, while the vibrational relaxation is four times slower, caused by the relatively weak intermolecular coupling between molecules. The results of classical all-atom simulations allow a parameter free description of the experimental results. The observed population of rapidly reorienting water molecules is likely a general feature of water near extended hydrophobic surfaces and is an important additional characteristic of how water meets such surfaces. ${ }^{8}$

\section{Conclusions}

We use ultrafast two-dimensional surface-specific vibrational spectroscopy to probe the interfacial energy dynamics of heavy water $\left(D_{2} 0\right)$ at the water-air interface. The measurements reveal the presence of surprisingly rapid energy transfer, both between hydrogen-bonded interfacial water molecules (intermolecular), and between 0-D groups sticking out from the water surface and those located on the same molecule, pointing towards the water bulk (intramolecular). Vibrational energy transfer occurs on sub-picosecond time scales and its rates and pathways can be quantified directly. The reorientation of interfacial water molecules is several times faster than their bulk counterparts, and is concluded to occur in a more diffusive manner than in bulk, where constraints due to hydrogen bonds causes the motion to be jump-like and therefore non-diffusive.

\section{References}

[1] H. J. Bakker and J. L. Skinner, Chem. Rev. (Washington, DC, U. S.) 110, 1498-1517 (2010).

[2] D. Laage and J. T. Hynes, Science 311, 832-835 (2006).

[3] J. Bredenbeck, A. Ghosh, H. K. Nienhuys and M. Bonn, Acc. Chem. Res. 42, 1332-1342 (2009).

[4] J. Bredenbeck, A. Ghosh, M. Smits and M. Bonn, J. Am. Chem. Soc. 130, 2152-+ (2008).

[5] Z. Zhang, L. Piatkowski, H. J. Bakker and M. Bonn, Nature Chem. 3, 888-893 (2011).

[6] Z. Zhang, L. Piatkowski, H. J. Bakker and M. Bonn, J. Chem. Phys. 135021101 (2011).

[7] C. S. Hsieh, R. K. Campen, A. C. V. Verde, P. Bolhuis, H. K. Nienhuys and M. Bonn, Phys. Rev. Lett. 107116102 (2011).

[8] G. Stirnemann, P. J. Rossky, J. T. Hynes and D. Laage, Faraday Discuss. 146, 263-281 (2010). 